\title{
Enfermedades genéticas del ADN mitocondrial humano
}

\author{
A belardo Solano, Q . F. B., ${ }^{(1)}$ A na Playán, Ph.D., ${ }^{(1)}$ \\ Manuel J. López-Pérez, Ph.D., ${ }^{(1)}$ Julio Montoya, Ph.D. ${ }^{(1)}$
}

\section{Solano A, Playán A, López-Pérez MJ, Montoya J. Enfermedades genéticas del ADN mitocondrial humano.} Salud Publica Mex 2001;43:151-161. EI texto completo en inglés de este artículo está disponible en: http://www.insp.mx/salud/index.html

\section{Resumen}

Las enfermedades mitocondriales son un grupo de trastornos que están producidos por un fallo en el sistema de fosforilación oxidativa (sistema 0 xphos), la ruta final del metabolismo energético mitocondrial, con la consiguiente deficiencia en la biosíntesis del trifosfato de adenosina (ATP, por sus siglas en inglés). Parte de los polipéptidos que componen este sistema están codificados en el ácido desoxirribonucleico (DN A) mitocondrial y, en los últimos años, se han descrito mutaciones que se han asociado con síndromes clínicos bien definidos. Las características genéticas del D N A mito condrial, herencia materna, poliplasmia y segregación mitótica, confieren a estas enfermedades propiedades muy particulares. Las manifestaciones clínicas de estas enfermedades son muy heterogéneas y afectan a distintos órganos y tejidos por lo que su correcto diagnóstico implica la obtención de datos clínicos, morfológicos, bioquímicos y genéticos. El texto completo en inglés de este artículo está disponible en: http://www.insp.mx/salud/ index.html

Palabras clave: ADN mitocóndrico; enfermedades mitocondriales; España

\author{
Solano A, Playán A, López-Pérez MJ, Montoya J. \\ Genetic diseases \\ of the mitochondrial DNA. \\ Salud Publica Mex 2001;43:151-161. \\ The English version of this paper \\ is available at: http://www.insp.mx/salud/index.html
}

\begin{abstract}
A bstract
Mitochondrial diseases are a group of disorders produced by defects in the oxidative phosphorylation system $10 \mathrm{x}$ phos system), the final pathway of the mitochondrial energetic metabolism, resulting in a deficiency of the biosynthesis of ATP. Part of the polypeptide subunits involved in the 0 xphos system are codified by the mitochondrial DNA. In the last years, mutations in this genetic system have been described and associated to well defined clinical syndromes. The clinical features of these disorders are very heterogeneous affecting, in most cases, to different organs and tissues and their correct diagnosis require precise clinical, morphological, biochemical and genetic data. The peculiar genetic characteristics of the mitochondrial DNA (maternal inheritance, polyplasmia and mitotic segregation) give to these disorders very distinctive properties. The English version of this paper is available at: http://www.insp.mx/salud/ index.html
\end{abstract}

Key words: DNA, mitochondrial; mitochondrial diseases; Spain
T as mitocondrias son organelos subcelulares que se $\perp$ encuentran en el citoplasma de las células eucariotas, cuya función principal es la producción de la energía celular en forma de trifosfato de adenosina
(ATP, por sus siglas en inglés). Una de las particularidades de estos organelos es la de poseer un sistema genético propio con toda la maquinaria necesaria para su expresión, es decir, para replicar, transcribir y

Este trabajo ha sido subvencionado por la Dirección General de Enseñanza Superior e Investigación Científica (PB97-1019), el Fondo de Investigaciones Sanitarias (FIS 98-0049-01), la Diputación General de Aragón (P24/97) de España, así como por el Consejo N acional de Ciencia y Tecnología (Conacyt) de México.

(1) Departamento de Bioquímica y Biología Molecular y Celular, Universidad de Zaragoza, Zaragoza, España.

Fecha de recibido: 22 de mayo de 2000 - Fecha de aprobado: 13 de noviembre de 2000

Solicitud de sobretiros: Dr. Julio Montoya. Departamento de Bioquímica y Biología Molecular y Celular, Facultad de Veterinaria, Universidad de Zaragoza. Miguel Servet 177, E-50013 Zaragoza, España.

Correo electrónico: jmontoya@ posta.unizar.es 
traducir la información genética que contiene. El ácido desoxirribonucleico mitocondrial (mtDNA, por sus siglas en inglés) humano es una molécula circular compuesta por 16569 pares de bases ${ }^{1}$ que contiene información para 37 genes: dos ácidos ribonucleicos ribosómicos (rRNA), componentes de los ribosomas específicos mitocondriales, 22 de transferencia (tRNA), que son capaces de leer todo el código genético, y 13 polipéptidos que forman parte de cuatro de los cinco complejos multienzimáticos del sistema de fosforilación oxidativa (sistema Oxphos), etapa terminal de la ruta de producción de ATP. Estos péptidos corresponden a siete subunidades (ND1, 2, 3, 4, 4L, 5, 6) del dinucleótido de nicotinamida y adenina reducido (NADH): ubiquinona óxido-reductasa (complejo I); una subunidad (cyt b) de la ubiquinol: citocromo $\mathrm{c}$ óxido-reductasa (complejo III); tres subunidades (CO I, II, III) de la citocromo c oxidasa (complejo IV), y dos subunidades de la ATP sintetasa (complejo V) ${ }^{2}$



Figura 1. Mapa genético del DNA mitocondrial HUMANO. Se REPRESENTA LAS DOS HEBRAS DEL DNA CON LOS GENES QUE CODIFICAN: RRNA (12S Y 16S), TRNA, SEÑALADOS CON LA ABREVIATURA DEL AMINOÁCIDO QUE TRANSPORTAN, Y SECUENCIAS CODIFICADORAS DE PROTEINAS (CO: SUBUNIDADES CITOCROMO C OXIDASA; CYT B: CITOCROMO B Y ND: SUBUNIDADES DE NADH DESHIDROGENASA). $H_{1}, H_{2} Y$ L INDICAN LOS LUGARES DE INICIACIÓN DE LA TRANSCRIPCIÓN DE LAS HEBRAS PESADA Y LIGERA, RESPECTIVAMENTE. $\boldsymbol{O}_{\mathrm{H}}$ Y $\mathbf{O}_{\mathrm{L}}$ SIMBOLIZAN LOS ORíGENES DE REPLICACIÓN DE LA CADENA PESADA Y LIGERA (figura 1). El resto de los polipéptidos componentes de estos complejos, así como el complejo II completo, están codificados en el DNA nuclear. La biogénesis de este sistema constituye un caso único en la célula ya que para su formación se requiere la expresión coordinada de los dos sistemas genéticos.

Los caracteres moleculares básicos y peculiares del sistema genético mitocondrial se descubrieron al inicio de los años ochenta, ${ }^{1,-6}$ y en 1988 se encontraron las primeras mutaciones asociadas a enfermedades. ${ }^{7-9}$ Desde entonces, el número de mutaciones en el mtDNA y de enfermedades asociadas ha crecido de modo espectacular y ha generado lo que hoy se podría llamar como una "medicina mitocondrial". ${ }^{10,11}$

Se designa con el nombre de enfermedades mitocondriales a un grupo de trastornos cuya característica común es un defecto en la producción de ATP. Sin embargo, frecuentemente este término se aplica a trastornos producidos por daños en el sistema Oxphos, debido a que durante muchos años sólo se habían encontrado mutaciones en el mtDNA relacionados con los mismos. Hoy en día, se han comenzado a identificar genes nucleares codificantes de proteínas de los complejos del sistema Oxphos o responsables de su ensamblaje. En este trabajo nos limitaremos a describir las enfermedades debidas a daños en el sistema genético mitocondrial por ser las más conocidas y por presentar un modo de herencia muy particular.

\section{Caracteres específicos de la genética mitocondrial}

El tipo de herencia del sistema genético mitocondrial, su localización en un organelo citoplasmático, la disposición contínua de los genes sin nucleótidos intermedios ni intrones y la poliplasmia (alto número de copias en cada célula) proporcionan caracteres genéticos que los diferencian claramente de los del DNA nuclear. Cada célula contiene entre unas 1000 y 10000 copias de mtDNA dependiendo del tejido, pasando por unos cuantos cientos en los espermatozoides y hasta unas 100000 en el oocito. Cada mitocondria contiene entre 2 y 10 moléculas.

Herencia materna. El mtDNA se hereda por vía materna con un patrón vertical no mendeliano. La madre trasmite su genoma mitocondrial a todos sus hijos, pero solamente las hijas lo pasarán a todos los miembros de la siguiente generación y así sucesivamente. Esto se debe al elevado número de moléculas de mtDNA que existe en los óvulos (entre 100000 y 200000 copias) en comparación con unos pocos cientos que hay en los espermatozoides. Además, las mitocondrias 
que puedan entrar en el óvulo fecundado se eliminan por un proceso activo. ${ }^{12}$

Segregación mitótica. El fenotipo de una línea celular puede variar durante la división celular debido a que las mitocondrias se distribuyen al azar entre las células hijas por lo que si en una célula coexisten dos poblaciones de mtDNA, una normal y otra mutada (heteroplasmia), a lo largo de las divisiones se podrán originar tres genotipos diferentes: homoplásmico para el DNA mitocondrial normal, homoplásmico para el DNA mutado y heteroplásmico. Por tanto, el fenotipo de una célula con heteroplasmia dependerá del porcentaje de DNA mutado que contenga. Si el número de moléculas de mtDNA dañado es relativamente bajo se produce una complementación con las moléculas de DNA normal y no se manifestará el defecto genético. Cuando el DNA mutado sobrepasa un umbral determinado se manifestará un fenotipo patogénico (efecto umbral), es decir, si la producción de ATP llega a estar por debajo de los mínimos necesarios para el funcionamiento de los tejidos, debido a la producción defectuosa de proteínas codificadas en el mtDNA, se produce la aparición de la enfermedad. El número de moléculas de DNA es diferente en cada órgano y tejido según la cantidad de energía requerida para su funcionamiento. Por ello, los tejidos que preferentemente se afectan son la visión, el sistema nervioso central, músculo esquelético, corazón, islotes pancreáticos, riñón e hígado.

Alta velocidad de mutación. El mtDNA presenta una tasa de mutación espontánea 10 veces superior a la del DNA nuclear. Este fenómeno puede estar causado porque en la mitocondria se producen continuamente radicales de oxígeno, como consecuencia de la oxidación final de los compuestos carbonados, que pueden dañar a un DNA que no está protegido por proteínas. Debido a este hecho, la variación de secuencias entre individuos de una misma especie es muy grande, hasta unos 70 nucleótidos, ${ }^{13} \mathrm{y}$ en un mismo individuo se estará generando, a lo largo de la vida, una pequeña heterogeneidad en el mtDNA. De este modo, se ha llegado a proponer que la disminución en la capacidad respiratoria de los tejidos que tiene lugar en el envejecimiento pueda ser debida a una acumulación de este daño mitocondrial. ${ }^{14}$ Esta teoría tiene su primera evidencia en un trabajo del grupo de Attardi, que documenta que las mitocondrias se deterioran con la edad como consecuencia de la acumulación de mutaciones. ${ }^{15}$ Las variaciones de secuencia existentes entre diferentes individuos han resultado muy útiles para estudios antropológicos, etnológicos y forenses, y es la base de la hipótesis de que el hombre desciende de una mujer que vivió en Africa hace unos 250000 años ("Eva mitocondrial"). ${ }^{16}$

\section{Enfermedades genéticas del DNA mitocondrial}

Las enfermedades originadas por daños en el genoma mitocondrial tienen en común el estar producidas por una deficiencia en la biosíntesis de ATP, ya que toda la información que contiene este DNA está dirigida a la síntesis de proteínas componentes del sistema Oxphos. Las manifestaciones de estas enfermedades son muy variadas y pueden afectar a todos los órganos y tejidos, ya que la síntesis de ATP se produce en todos ellos y a cualquier edad. Estas pueden presentar una serie de aspectos clínicos, morfológicos y bioquímicos muy concretos que dan lugar a síndromes bien caracterizados pero, en la mayor parte de los casos, principalmente en edad pediátrica, los síntomas son muy poco informativos y es sólo la presencia de anormalidades neurológicas, a veces acompañadas de aumento de ácido láctico y de otros síntomas clínicos secundarios que afectan a diversos órganos, lo que da alguna orientación en el diagnóstico de una enfermedad mitocondrial. ${ }^{17}$ Entre las manifestaciones clínicas más comunes se encuentran una o varias de las siguientes: desórdenes motores, accidentes cerebrovasculares, convulsiones, demencia, intolerancia al ejercicio, ptosis, oftalmoplejia, retinopatía pigmentaria, atrofia óptica, ceguera, sordera, cardiomiopatía, disfunciones hepáticas y pancreáticas, diabetes, defectos de crecimiento, anemia sideroblástica, pseudo obstrucción intestinal, nefropatías, acidosis metabólica y otras más secundarias.

La presencia de uno o más de estos síntomas requiere a continuación de un estudio morfológico, histoquímico y bioquímico para asegurar la naturaleza de estas enfermedades. Así, con mucha frecuencia se encuentran: fibras rojo-rasgadas (acumulación de mitocondrias anormales en tamaño y número) en biopsias musculares teñidas con tricromo de Gomori y fibras no reactivas a la tinción histoquímica de la citocromo c oxidasa; defectos en uno o varios complejos de la cadena respiratoria; y desarreglos metabólicos con elevación de lactato, piruvato o una aminoaciduria generalizada causados por una disfunción de la cadena respiratoria que conlleva un aumento de equivalentes reductores en la mitocondria y citoplasma, y una alteración del funcionamiento del ciclo de Krebs debido al exceso de NADH, lo que provoca una acumulación de piruvato y su posterior conversión a lactato que difunde a la sangre. Sin em- 
bargo, la ausencia de algunos de estos caracteres no debe descartar la posibilidad de enfermedad mitocondrial, especialmente en pacientes en edad pediátrica. Además, los estudios familiares pueden ser decisivos si se comprueba la existencia de herencia materna de la enfermedad. El estudio genético del paciente y familiares relacionados por vía materna pueden asegurar finalmente que nos encontramos ante este tipo de trastornos. De hecho, hoy en día, el desarrollo y rapidez de las técnicas de genética molecular permiten, en ocasiones, una confirmación de la enfemedad antes de haber realizado muchas de las pruebas anteriormente citadas. La complejidad del diagnóstico de estas enfermedades hace preciso que los pacientes tengan que acudir a centros muy especializados donde se pueda llevar a cabo evaluaciones clínicas, metabólicas, patológicas, bioquímicas y genéticas, y a que en su diagnóstico estén implicados especialistas de muy diverso origen.

Desde que en 1988 se describieran las primeras enfermedades causadas por daños en el mtDNA, ${ }^{7-9}$ se han encontrado más de 150 mutaciones (más 100 deleciones y unas 50 mutaciones puntuales) asociadas a enfermedades humanas. El interés por su estudio ha crecido enormemente debido al gran aumento de pacientes diagnosticados con estos trastornos y a que se presentan desde en recién nacidos hasta en adultos de todas las edades. Además, muchas de estas mutaciones se trasmiten por línea materna, como se ha indicado anteriormente, lo que hace que el diagnóstico en un individuo pueda tener implicaciones en muchas generaciones de una familia.

A pesar de la importancia que las enfermedades mitocondriales tienen últimamente y de ser responsables de una considerable morbilidad, hasta ahora no se han realizado estudios exhaustivos sobre su prevalencia en la población general. Las razones son múltiples: ${ }^{18}$ complejidad de las manifestaciones clínicas, necesidad de biopsias musculares para su diagnóstico (no siempre se pueden detectar las mutaciones en muestras de sangre); necesidad de secuenciar todo el genoma mitocondrial para poder localizar mutaciones no detectadas hasta ahora, problemas éticos para realizar análisis genéticos presintomáticos en niños, diagnóstico erróneo de muchos pacientes al no ser atendidos en centros especializados, etcétera. Sin embargo, a pesar de todas estas dificultades, el grupo del doctor Turnbull, en Newcastle, Reino Unido, ha publicado muy recientemente los primeros datos epidemiológicos de las enfermedades del mtDNA, centrados en la población blanca de Europa del Norte residente en el noreste de Inglaterra. ${ }^{18}$ Así, ha mostrado que los defectos en el mtDNA son la causa de enfermedad en 6.57 de cada 100000 individuos de la población adulta trabajadora y que 7.59 por cada 100000 adultos y niños no afectados corren el riesgo de desarrollar una de estas enfermedades. En total, 12.48 por 100000 individuos (1 de cada 8 000) tienen o presentan un riesgo de padecer una enfermedad causada por daños en el mtDNA. Estos datos representan un mínimo de prevalencia porque, muy probablemente, el número de pacientes que han quedado sin diagnosticar es elevado por haber sido atendidos por médicos de asistencia primaria, y no en clínicas neurológicas, y que hayan podido pasar desapercibidos por presentar solamente algunos de los síntomas acompañantes de estas enfermedades como diabetes o ptosis.

Los datos obtenidos por el grupo de Newcastle han permitido comprobar que la prevalencia de las enfermedades debidas a daños en el mtDNA, consideradas en su conjunto, es equivalente a la de otras enfermedades neurológicas como la enfermedad de Huntington y la esclerosis amiotrófica lateral $(6.4 \mathrm{y}$ 6.2 por cada 100000 individuos, respectivamente), y superior a la de otras enfermedades neuromusculares hereditarias como la distrofia de Duchenne (3.2 por cada 100000 individuos). ${ }^{18}$

La experiencia de nuestro servicio de diagnóstico en la Universidad de Zaragoza es que un 16\% de los pacientes remitidos para el estudio genético presentan una deleción o mutación puntual. ${ }^{19,20, *}$ No hemos realizado ningún estudio de lo que este número representa entre la población general española; pero, seguro que tanto en el estudio realizado en Inglaterra como en nuestro laboratorio, el número de pacientes será muy superior cuando se secuencie el mtDNA de todos los posibles implicados y se detecten nuevas mutaciones. Estos números, junto al hecho de que no exista una terapia eficaz, y que, aunque algunas de estas enfermedades puedan mejorar o estabilizarse a lo largo de su curso, ilustran la importancia que tienen en relación con la salud pública, particularmente en cuanto a su atención y consejo genético, pues la mayoría tiene un desenlace fatal.

La heterogeneidad de las manifestaciones clínicas, morfológicas y bioquímicas de las enfermedades del mtDNA, hace que su clasificación se base muy frecuentemente en las características genéticas de las mutaciones, a pesar de que, en algunos casos, una misma mutación pueda dar lugar a fenotipos clínicos muy diversos. Así, las enfermedades del mtDNA

\footnotetext{
* Solano A. Enfermedades del mtDNA (tesis doctoral). Zaragoza Universidad de Zaragoza. En realización, 2000.
} 
se pueden dividir en tres grandes grupos según estén asociadas a mutaciones puntuales, a reorganizaciones o a disminución de número de copias del mtDNA. En la severidad de la manifestación de la enfermedad intervienen varios factores: la naturaleza de la mutación, el grado de heteroplasmia, los requerimientos energéticos del tejido y la capacidad del tejido para compensar el daño celular. A continuación se presenta un resumen de las enfermedades más comunes asociadas a estos tipos de mutaciones.

\section{Enfermedades asociadas a mutaciones puntuales en el $m t D N A$}

Dado el alto índice de mutación del mtDNA, como se ha indicado anteriormente, es posible encontrar un gran número de mutaciones puntuales. Sin embargo, la mayoría son mutaciones silenciosas que no causan ningún tipo de defecto. Las mutaciones patológicas se pueden encontrar tanto en los genes de tRNA, de rRNA, como en los codificantes de proteínas, y responden siempre a un tipo de herencia materna.

Neuropatía óptica hereditaria de Leber. La neuropatía óptica hereditaria de Leber (LHON) se caracteriza por la pérdida bilateral de la visión central, originada por atrofia del nervio óptico. Aparece en la segunda o tercera década de la vida y afecta a más hombres que a mujeres. Aunque normalmente sólo la visión está afectada, hay casos en los que también aparecen trastornos en la conducción cardiaca, neuropatía periférica y ataxia cerebelar.

Esta fue la primera enfermedad humana de herencia materna que se asoció a una mutación en el mtDNA. Después se llegó a asociar hasta con 16 mutaciones puntuales (cuadro I), localizadas todas ellas en genes codificantes de proteínas, y que se clasificaron en primarias, secundarias o intermedias según su relación con la aparición de la enfermedad. Sin embargo, últimamente sólo tres, G3 460A, G11 778A y T14 484C, están consideradas como primarias o patogénicas verdaderas, siendo la G11 778A la responsable en $50 \%$ de los casos y la que provoca la forma más severa de la enfermedad. Las tres se encuentran en genes que codifican algún polipéptido del complejo I del sistema Oxphos. La detección de estas mutaciones se suele hacer en células sanguíneas donde se encuentran tanto en forma homo como heteroplásmica. El resto de las mutaciones se consideran como secundarias, suelen acompañar a las anteriores en forma homoplásmica y se desconoce su relación directa con la enfermedad. Entre estas últimas vale la pena mencionar que la mutación G15257A, consi- derada como intermedia por algunos autores, se ha encontrado en varias familias analizadas en nuestro laboratorio por lo que pensamos que puede contribuir de forma decisiva a la aparición de la enfermedad.

La prevalencia de la enfermedad en hombres ha sugerido la influencia de un gen nuclear, y aunque se ha descrito un ligamiento de la enfermedad con el locus (DXS7) situado en el cromosoma X en familias finlandesas, ${ }^{21}$ no se ha podido confirmar en familias de otro origen.

Síndrome de neuropatía, ataxia y retinopatía pigmentaria. Este síndrome está caracterizado por debilidad muscular neurogénica, ataxia y retinitis pigmentosa. Suele ir acompañado de demencia, convulsiones y neuropatía sensorial axonal, presenta una herencia materna y se ha asociado a una mutación puntual, T8993G, en el gen de la subunidad 6 de la ATPasa (cuadro I). La mutación aparece normalmente en forma heteroplásmica y en todos los tejidos estudiados: leucocitos, fibroblastos, músculo, riñón y cerebro. Existe una alta correlación entre la proporción del DNA mutado y la severidad de la enfermedad.

Síndrome de Leigh de herencia materna. El síndrome de Leigh de herencia materna (MILS) es una enfermedad muy heterogénea que se puede presentar asociada a diferentes tipos de herencia, autosómica recesiva, ligada al cromosoma $\mathrm{X}$ o materna (mitocondrial) según el gen que esté dañado. Es una enfermedad devastadora que se caracteriza por trastornos degenerativos multisistémicos que aparecen en el primer año de vida, disfunciones del tallo cerebral y de los ganglios basales, desmielinización, regresión psicomotora, retraso en el desarrollo, ataxia, convulsiones, neuropatía periférica. El diagnóstico se confirma por la presencia de lesiones necróticas cerebrales focales en el tálamo, tallo cerebral y núcleo dentado. La forma de la enfermedad, que se hereda por vía materna, está producida por la mutación en el gen de la subunidad 6 de la ATPasa, T8993G, la misma que produce el síndrome de neuropatía, ataxia y retinopatía pigmentaria, pero con un porcentaje de la mutación superior a $90 \%$. Otras formas menos severas de esta enfermedad se han asociado con un cambio $\mathrm{T} \varnothing \mathrm{C}^{*}$ en la misma posición del mtDNA.

Síndrome de epilepsia mioclónica con fibras rojo-rasgadas (MERRF). Este síndrome de herencia materna, está caracterizado por epilepsia mioclónica, convulsiones generalizadas y miopatía con presencia de fibras rojorasgadas. Otros síntomas clínicos que pueden acompañar a los anteriores son demencia, sordera, neuropatía,

* Timina por citosina. 
atrofia óptica, fallo respiratorio y cardiomiopatía. Aparece tanto en la infancia como en edad adulta y es de curso progresivo. Está asociado a la presencia de mutaciones en el gen del mtDNA para el tRNA ${ }^{\text {Lys. En la }}$ mayoría de los casos (80\%-90\%) se debe a una mutación A8344G, pero también se han encontrado otras mi- noritarias como T8356C (cuadro I), todas en forma heteroplásmica. El porcentaje de heteroplasmia necesario para la afectación varía entre individuos jóvenes (95\%) e individuos por encima de los 60-70 años $(60 \%)$ del DNA mutado. ${ }^{13}$ La presencia de estas mutaciones en tRNA daña la síntesis de proteínas.

\section{Cuadro I}

\section{Mutaciones en el MtDNA y enfermedades ASOCIADAS}

\begin{tabular}{|c|c|c|c|c|c|c|c|}
\hline Enfermedad & $\begin{array}{c}\text { Mutación } \\
\text { en el mt } D N A\end{array}$ & $\begin{array}{c}\text { Gen } \\
\text { afectado }\end{array}$ & Referencias & Enfermedad & $\begin{array}{c}\text { Mutación } \\
\text { en el mtDNA }\end{array}$ & $\begin{array}{c}\text { Gen } \\
\text { afectado }\end{array}$ & Referencias \\
\hline LHON & & & & Miopatía mitocondrial & T3250C & tRN A Leu(uur) & 53 \\
\hline \multirow[t]{3}{*}{ Mutaciones primarias } & G3460A & ND1 & 22,23 & & A3302G & tRN $A^{\text {Leu(uur) }}$ & 54 \\
\hline & G11778A & ND4 & 7 & & C15990T & tRN A Pro & 55 \\
\hline & T14484C & ND6 & 24 & Sordera inducida por & & & \\
\hline \multirow[t]{2}{*}{ Mutaciones intermedias } & G5244A & ND2 & 25 & aminoglicósidos & A1555G & $12 \mathrm{~S}$ rRN A & 56 \\
\hline & G15257A & Citocromo b & 25 & Sordera sensoneural & T7445C & 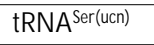 & 57 \\
\hline \multirow[t]{12}{*}{ Mutaciones secundarias } & T3394C & ND1 & 24 & Anemia sideroblástica & G12301A & tRN $A^{\text {Leu(cun) }}$ & 58 \\
\hline & T4160C & ND1 & 26 & Lipomatosis múltiple & & & \\
\hline & T4216C & ND1 & 27 & simétrica & A8344G & tRN A Lys & 59 \\
\hline & A4917G & ND2 & 27 & CPEO & Deleción única & & 60 \\
\hline & G7444A & $\mathrm{COI}$ & 28 & & A3243G & tRN $A^{\text {Leu(uur) }}$ & 61 \\
\hline & T9101C & ATPasa 6 & 29 & & A5692G & tRN A ${ }^{\text {Asn }}$ & 62 \\
\hline & G9438A & CO III & 30 & & G5703A & tRN $A^{\text {Asn }}$ & 63 \\
\hline & G9804A & CO III & 30 & & C 3256T & tRN $A^{\text {Leu(uur) }}$ & 63 \\
\hline & G13708A & ND5 & 27 & Intolerancia al ejercicio & G 15084A & Citocromo b & 64 \\
\hline & G13730A & ND5 & 31 & & G15168A & Citocromo b & 64 \\
\hline & G14459A & ND6 & 32 & & G15723A & Citocromo b & 64 \\
\hline & G15812A & cyt b & 25 & & G 14846A & Citocromo b & 64 \\
\hline NARP & T8993G & ATPasa 6 & 33 & & delecion de $24 \mathrm{pb}$ & Citocromo b & 64 \\
\hline \multirow[t]{2}{*}{ Leigh (MILS) } & T8993G & ATPasa 6 & 34,35 & LIMM & A15923G & tRN $A^{\text {Thr }}$ & 65 \\
\hline & T8993C & ATPasa 6 & 36 & Muerte súbita & A3251G & tRN A Leu(uur) & 66 \\
\hline \multirow[t]{5}{*}{ MELAS } & A3243G & tRN A Leu(uur) $^{\text {Le }}$ & 37 & Necrosis bilateral & & & \\
\hline & C 3256T & tRN A Leu(uur) & 38 & del estriado & T9176C & ATPase 6 & 67 \\
\hline & T3271C & tRN A Leu(uur) & 39 & & $\mathrm{~T} 8851 \mathrm{C}$ & ATPase 6 & 68 \\
\hline & T3291C & tRN A $A^{\text {Leu(urr) }}$ & 40 & Multisistémicas & A3251G & tRN A $A^{\text {Leu(uur) }}$ & 66 \\
\hline & T9957C & COIII & 41 & & A3252G & tRN A Leu(uur) $^{\text {Lef }}$ & 69 \\
\hline \multirow[t]{2}{*}{ MERRF } & A8344G & tRN A Lys & 42 & & C $3256 \mathrm{~T}$ & tRN A Leu(uur) & 63 \\
\hline & T8356C & tRN A Lys & 43 & Corea y demencia & G5549A & tRN A $^{\text {Trp }}$ & 70 \\
\hline Diabetes y sordera & A3243G & tRN $A^{\text {Leu(uur) }}$ & 44 & LHON y distonía & G 14459A & ND6 & 32 \\
\hline \multirow[t]{8}{*}{ Cardiomiopatía (MICM) } & A3260G & tRN A Leu(urr) & 45 & Diabetes y miopatía & T14709C & ND6 & 71 \\
\hline & C3303T & tRN A $A^{\text {Leu(uur) }}$ & 46 & Pearson & Deleción única & & 72 \\
\hline & A4269G & tRN Alle & 47 & Kearns-Sayre & Deleción única & & 73 \\
\hline & A4300G & tRN Alle & 48 & & & & \\
\hline & A4317G & tRN Alle & 49 & & & & \\
\hline & C4320T & tRN Alle & 50 & & & & \\
\hline & G8363A & tRN A Lys & 51 & & & & \\
\hline & T9997C & tRN A Gly & 52 & & & & \\
\hline
\end{tabular}

LIMM: miopatía mitocondrial infantil letal; LHON : neuropatía óptica hereditaria de Leber; MELAS: encefalomiopatía mitocondrial con acidosis láctica y episodios de accidentes cerebrovasculares; MERRF: epilepsia mioclónica con fibras rojo-rasgadas; MICM: Cardiomiopatía de herencia materna; MILS: síndrome de Leigh de herencia materna; PEO : oftalmoplejia progresiva externa 
Síndrome de encefalomiopatía mitocondrial con acidosis láctica y episodios de accidentes cerebro-vasculares (MELAS). Se trata de una encefalomiopatía mitocondrial, de herencia materna, caracterizada por accidentes cerebrovasculares producidos a edad temprana que provocan una disfunción cerebral subaguda y cambios en la estructura cerebral, y por acidosis láctica. Estos caracteres suelen ir acompañados de convulsiones generalizadas, dolor de cabeza, sordera, demencia $y$, a veces, presenta fibras rojo-rasgadas.

Esta enfermedad ha sido asociada fundamentalmente con mutaciones en el gen del tRNA ${ }^{\text {Leu(UUR) }}$ del mtDNA. La mayor parte de los casos (80\%) está asociada a la mutación A3.243G, pero también se han encontrado otras con menor incidencia y alguna en genes codificantes de proteínas (cuadro I), todas en forma heteroplásmica. Al igual que en la epilepsia mioclónica, las mutaciones en el tRNA dañan la síntesis de proteínas mitocondriales.

Diabetes de herencia materna con sordera. Además de los dos tipos clásicos de diabetes dependiente y no dependiente de insulina (tipo 1 y 2 , respectivamente), se ha descrito recientemente un nuevo tipo de diabetes asociada a sordera, que no encuadra dentro de la clasificación de la Organización Mundial de la Salud. Esta diabetes, de herencia materna, está producida por la mutación A3.243G en el gen del tRNA ${ }^{\mathrm{Leu}(\mathrm{UUR})}$ (cuadro I), la misma descrita para el síndrome de (MELAS). La frecuencia de diabetes y sordera es aproximadamente de un $1.5 \%$ de la población diabética total. ${ }^{74}$ Por otra parte, la diabetes es una de las enfermedades que se han descrito asociadas a otros síndromes mitocondriales como la encefalomiopatía mitocondrial, oftalmoplejia progresiva externa crónica, KearnsSayre, Pearson y diabetes insípida, diabetes mellitus, atrofia óptica y sordera (DIDMOAD).

\section{Otras enfermedades del mtDNA asociadas a mutaciones puntuales}

Además de las enfermedades descritas anteriormente, hay otras muchas que se han asociado a otras mutaciones puntuales (cuadro I). Entre ellas, se pueden citar las cardiomiopatías de herencia materna relacionadas fundamentalmente con mutaciones en el tRNA ${ }^{\text {Ile: }}$ la sordera inducida por aminoglicósidos que está producida por una mutación en el rRNA 12S (A1555G), y otros tipos de sordera sindrómica o no sindrómica de herencia materna; LHON y distonía; miopatías de herencia materna unidas a mutaciones en $\mathrm{RNA}^{\mathrm{Leu}}$, $\mathrm{tRNA}^{\text {Pro, }}$, tRNA ${ }^{\mathrm{Asn}}$, $\mathrm{tRNA}^{\mathrm{Tyr}}$; oftalmoplejia progresiva externa crónica; anemia sideroblástica; deficiencia fatal de la cadena respiratoria infantil; lipomatosis si- métrica múltiple asociada a la mutación A8.344G del gen del tRNA ${ }^{\text {Lys }}$ (descrita en nuestro laboratorio) y, recientemente, se ha relacionado la intolerancia al ejercicio, como entidad propia, a mutaciones puntuales en el gen del citocromo b. Así, se han descrito mutaciones en este gen que crean un codón de terminación, que cambian un aminoácido o, incluso, una deleción de 24 pares de bases. En el cuadro I se citan éstos y otros síndromes que se han asociado a mutaciones puntuales y, sin ninguna duda, el espectro de fenotipos relacionados con mutaciones en el mtDNA aumentará más en un futuro. Asimismo, cabe mencionar que alguna de las mutaciones, como la A3.243G, puede estar relacionada con muy diversos fenotipos clínicos como sindromes de encefalopatía mitocondrial con acidosis láctica y episodios de accidentes cerebrovasculares de epilepsia mioclónica con fibras rojo-rasgadas y solapados, cardiomiopatías, CPEO, etcétera. Actualmente, se estudia la posible implicación del mtDNA en enfermedades neurodegenerativas como Parkinson y Alzheimer.

\section{Enfermedades asociadas a reorganizaciones en el DNA mitocondrial}

Además de las mutaciones puntuales, el mtDNA puede sufrir otro tipo de daños como son la pérdida de parte del mismo (deleciones) o la adición de un nuevo fragmento del DNA (duplicaciones), que, como en los casos anteriores, afectan a la biogénesis del sistema Oxphos y, por tanto, a la síntesis de ATP. En la actualidad hay descritos más de 100 tipos de deleciones y sólo unos cuantos casos de inserciones. Este tipo de mutaciones suelen ser espontáneas, probablemente causadas por daños en genes nucleares que controlan la replicación del $\mathrm{mtDNA}$, aunque hay descritos casos de herencia materna. ${ }^{75}$ Se presentan siempre en forma heteroplásmica, ya que la homoplasmia sería incompatible con la vida, y se sabe que la gravedad de los casos aumenta con la edad debido a la ventaja replicativa de estas moléculas de DNA más pequeñas en relación con la de tamaño normal.

Los tres tipos de síndromes más comunes en los que se presentan deleciones son los de Pearson, oftalmoplejia progresiva externa crónica y Kearns-Sayre.

Síndrome de médula ósea-páncreas de Pearson. Es una enfermedad que aparece en los primeros años de vida y que afecta a la hematopoyesis y a la función pancreática exocrina. Las características clínicas más comunes son anemia sideroblástica con vacuolización de precursores de la médula ósea que se manifiesta con una anemia macrocítica, trombocitopenia y neutropenia. 
Los niños afectados suelen morir antes de los tres años de edad y los que sobreviven suelen desarrollar posteriormente el fenotipo de Kearns-Sayre, que veremos más adelante. Estos pacientes presentan deleciones grandes únicas del mtDNA, en general son esporádicas aunque se ha descrito algún caso de herencia materna.

Oftalmoplejia progresiva externa crónica. Esta enfermedad está caracterizada por oftalmoplejia, ptosis bilateral de los párpados y miopatía. Suele ir acompañada también de intolerancia al ejercicio y debilidad muscular. En el músculo se encuentran fibras rojo-rasgadas COX negativas. En general, es una enfermedad benigna que suele aparecer en la adolescencia o en adultos jóvenes. Aparece de forma esporádica sin historia familiar. Se ha asociado fundamentalmente a deleciones grandes y únicas en el mtDNA (ver más adelante). Asimismo, se han encontrado otras formas de CPEO con mutaciones puntuales de herencia materna (cuadro I) o con deleciones múltiples de herencia autosómica recesiva o dominante.

Síndrome de Kearns-Sayre. Este síndrome es, por otra parte, una enfermedad multisistémica progresiva caracterizada clinicamente por $\mathrm{CPEO}$, retinopatía pigmentaria atípica, ataxia, miopatía mitocondrial, bloqueo de la conducción cardiaca, elevados niveles de proteína CSF (fluido cerebro espinal, por sus siglas en inglés), sordera y demencia. Aparece antes de los 20 años de edad.

Estas tres enfermedades están causadas por deleciones (de 2 a $9 \mathrm{~kb}$ ) en el mtDNA que suelen aparecer de forma espontánea. En general, la deleción es única, pero también se han descrito casos de deleciones múltiples. La gravedad de la enfermedad depende del porcentaje de DNA mutado en el individuo. En general están localizadas en el arco grande comprendido entre los orígenes de replicación del DNA y mantienen siempre las secuencias requeridas para la replicación del DNA y los promotores de la transcripción. Entre todas las deleciones conocidas, hay una que aparece con más frecuencia (hasta en 50\%), la llamada deleción común, que elimina un tramo de DNA de 4977 pares de bases (entre los nucleótidos 8483 a 13 460), que comprende los genes localizados entre la subunidad 8 de la ATPasa y ND5 (figura 1). No existe una clara relación entre el fenotipo y el tipo, tamaño o porcentaje del DNA delecionado ya que la misma deleción puede dar lugar a varios fenotipos diferentes. La mayor parte de las deleciones encontradas están flanqueadas por repeticiones directas de longitud variable (3-13 nt). Este hecho sugiere que la delección se produce por errores sucedidos en el proceso de replica- ción dependientes de la presencia de estas repeticiones. La pérdida de genes, especialmente la de los tRNA, hace que estos genomas no se puedan traducir $y$, por tanto, que sean dependientes de complementación con moléculas de mtDNA normales en la misma mitocondria. El umbral se suele alcanzar cuando el porcentaje de moléculas delecionadas supera $60 \%$.

Existen otras enfermedades como una diabetes con sordera y atrofia óptica; miopatías en general; el síndrome de encefalomiopatía mitocondrial neurogastrointestinal; el de diabetes mellitus, diabetes insípida, atrofia óptica y sordera, etcétera, que están asociadas a la presencia de deleciones en el mtDNA.

Como se ha mencionado anteriormente, entre las reorganizaciones del mtDNA se pueden encontrar duplicaciones en pacientes con defectos en el sistema Oxphos. Estas pueden ser también esporádicas o de herencia materna. Se han encontrado en pacientes de Kearns-Sayre, Pearson, diabetes mellitus, tubulopatía renal y miopatía mitocondrial e incluso en individuos normales. El mecanismo por el cual pueden causar la patogenicidad no está nada claro todavía.

\section{Enfermedades asociadas a depleciones de DNA mitocondrial}

El tercer tipo de daños en el genoma mitocondrial que puede causar enfermedades no se debe a mutaciones propiamente dichas sino a una disminución de los niveles del mtDNA. El espectro clínico que produce la depleción es muy variado. Los casos descritos hasta ahora afectan fundamentalmente a niños con combinaciones variables de miopatía, nefropatía o hepatopatía, miopatía infantil fatal por fallo respiratorio y algún otro con implicación multisistémica. La depleción puede estar producida por mutaciones en genes nucleares que controlan el número de copias del mtDNA. Es, por tanto, un trastorno de herencia mendeliana que afecta a la coordinación núcleo-mitocondria, y que parece ser autosómico recesivo.

\section{Regreso a la genética mendeliana}

Debido al doble origen genético nuclear y mitocondrial del sistema Oxphos las enfermedades genéticas mitocondriales pueden estar originadas, además de por mutaciones en genes del mtDNA con herencia materna, como ya hemos visto, por mutaciones en genes nucleares que codifican proteínas mitocondriales, por mutaciones que afecten al procesamiento postraduccional, al importe de proteínas por la mitocondria y al ensamblaje de los complejos, y por mutaciones que afecten al control nuclear del genoma 
mitocondrial, todas ellas con un tipo de herencia mendeliana.

El primer caso ha sido hasta ahora el más estudiado por estar el genoma mitocondrial completamente secuenciado y, por tanto, por la facilidad de encontrar mutaciones que afecten a los genes que codifica. Sin embargo, la mayor parte de los genes que componen el sistema Oxphos es de origen nuclear y cabe esperar que la mayoría de las enfermedades causadas por la deficiencia de este sistema sean debidas a mutaciones en el DNA nuclear. Así, por ejemplo, a pesar de que una de las causas del síndrome de Leigh sea una mutación en el mtDNA de herencia materna, se sabe que se transmite con más frecuencia por herencia autosómica recesiva, y se han encontrado e identificado mutaciones en genes de subunidades del complejo I, codificadas por el DNA nuclear ${ }^{76,77} \mathrm{y}$ en el complejo $\mathrm{II}^{78}$ codificado enteramente en el núcleo. Asimismo, se han localizado mutaciones en un gen nuclear (SURF 1) que codifica una proteína que, aunque no forma parte del complejo IV, es necesaria para su ensamblaje. ${ }^{79,80}$

Además, en la mitocondria existen muchas otras rutas metabólicas en las que no participa para nada el mtDNA y cuya deficiencia puede causar encefalomiopatías mitocondriales. Por todo ello, la genética mendeliana de las enfermedades mitocondriales está todavía prácticamente por descubrir y nos proporcionará mucha información sobre estos trastornos.

\section{Referencias}

1. Anderson S, Bankier AT, Barrell BG, de-Bruijn MHL, Coulson AR, D rouin J et al. Sequence and organization of the human mitochondrial genome. N ature 1981;290:427-465.

2. Chomyn A, Mariottini P, Cleeter MW J, Ragan Cl, D oolittle RF, MatsunoYagi $A$ et al. Functional assignment of the products of the unidentified reading frames of human mitochondrial DNA. En: Q uagliarello E, Slater EC, Plamieri F, Saccone C, Kroon AM, ed. Amsterdam: Elsevier Sciences, 1985:259-275.

3. Montoya J, 0 jala D, Attardi G. D istinctive features of the 5 '-terminal sequences of the human mitochondrial mRN As. N ature 1981; 290: 465-470.

4. O jala D, Montoya J, Attardi G. tRN A punctuation model of RN A processing in human mitochondria. $N$ ature 1981; 290:470-474.

5. Montoya J, Christianson T, Levens D, Rabinowitz M, Attardi G. Identification of initiation sites for heavy strand and light strand trascription in human mitochondrial DN A. Proc N atl A cad Sci USA 1982; 79:7195-7199. 6. Montoya J, Gaines GL, Attardi G. The pattern of transcription of the human mitochondrial rRNA genes reveals two overlaping transcription units. Cell 1983; 34:151-159.

7. W allace DC, Singh G, Lott MT, Hodge JA, Schurr TG, Lezza AMS et al. Mitochondrial DNA mutation associated with Leber's hereditary optic neuropathy. Science 1988; 242:1427-1430.
8. Holt IJ, Harding AE, Morgan-Hughes JA. D eletions of muscle mitochondrial DNA in patients with mitochondrial myopathies. Nature 1988; 331:717-719.

9. Zeviani M, Moraes CT, DiMauro S, Nakase H, Bonilla E, Schon E et al. Deletions of mitochondrial DNA in Kearns-Sayre syndrome. Neurology 1988; 38:1339-1346.

10. DiMauro S, Bonilla E. Mitochondrial encephalomyopathies. En: Rosenberg RN , Prusiner SB, DiMauro S, Barchi RL, ed. Boston: ButterworthHeinemann, 1998:201-235.

11. W allace DC. Mitochondrial DN A mutations and bioenergetic defects in aging and degenerative diseases. En: Rosenberg RN, Prusiner SB, DiMauro S, . Barchi RL, ed. Boston: Butterworth-H einemann, 1998:237- 269. 12. Sutovsky P, Moreno RD Schatten G. Ubiquitin tag for sperm mitochondria. N ature 1999; 402:371.

13. W allace DC. Diseases of the mitochondrial DNA. Annu Rev Biochem 1992; 61:1175-1212.

14. Miquel J. An update of the oxygen stress-mitochondrial mutation theory of aging: Genetic and evolutionary implications. Exp Gerontol 1998; 33:113-126.

15. Michikawa Y, Mazzucchelli F, Bresolin N, Scarlato G, Attardi G. Agingdependent large accumulation of point mutations in the human mtD N A control region for replication. Science 1999; 286:774-779.

16 Cann RL, Stoneking M, W ilson AC. Mitochondrial DNA and human evolution. N ature 1987; 325:31-36.

17. Munnich A, Rotig A, Chretien D, Cormier V, Bourgeron T, Bonnefont JP et al. Clinical presentation of mitochondrial disorders in childhood. J Inherited Metab Dis 1996;19:521-527.

18. Chinnery PF, Johnson MA, W ardell TM, SinghKler R, Hayes C, Brown DT et al. The epidemiology of pathogenic mitochondrial DNA mutations. Ann N eurol 2000; 48:188-193.

19. Beltrán B. A nálisis molecular de enfermedades del DN A mitocondrial: aplicación al diagnóstico (tesis doctoral). Zaragoza: Universidad de Zaragoza, 1995.

20. Playán A. Diagnóstico molecular de enfermedades del DNA mitocondrial (tesis D octoral). Zaragoza: Universidad de Zaragoza, 1999.

21. Vilkki J, 0 tt J, Savontaus ML, A ula P, N ikoskelainen EK. 0 ptic atrophy in Leber hereditary optic neuroretinopathy is probably determined by an X-chromosomal gene closely linked to DX S7. Am J Hum Genet 1991; 48:486-491.

22. Howell N, Bindoff LA, Mccullough DA, Kubaccka I, Poulton J, Mackey $D$ et al. Leber hereditary optic neuropathy: Identification of the same mitochondrial N DI mutation in six pedigrees. Am J Hum Genet 1991;49: 939-950.

23. Houponen K, Vilkki J, Aula P, N ikoskelainen EK, Savontaus ML. A new mitochondrial DN A mutation associated with Leber hereditary optic neuropathy. Am J Hum G enet 1991; 48:1147-1153.

24. Johns DR, N eufeld MJ, Park RD. An N D-6 mitochondrial DN A mutation associated with Leber hereditary optic neurtopathy. Biochem Biophys Res Commun 1992; 187:1551-1557.

25. Brown MD, Voljavec AS, Lott MT, Torrini A, Yang CC, W allace DC. Mitochondrial DNA complex I and III mutations associated with Leber's hereditary optic neuropathy. Genetics 1992; 130:163-173.

26. Howell N, Kubacka I, Xu M, McC ullogh DA. Leber hereditary optic neuropathy: Involvement of the mitochondrial NDI gene and evidence for an intragenic suppressor mutation. Am J Hum Genet 1991; 48:935-942.

27. Johns DR, Berman J. Alternative, simultaneous complex I mitochondrial DNA mutations in Leber's hereditary optic neuropathy. Biochem Biophys Res Commun 1991; 174:1324-1330.

28. Brown MD, Yang C, Trounce I, Torroni A, Lott MT, W allace DC. A mitochondrial DNA variant, identified in Leber hereditary optic neuropathy patients, which extends the aminoacid sequence of cytochrome c oxidase subunit 1. Am J HumG enet 1992; 51:378-385.

29. Lamminen T, Majander A, Juvonen V, W ikstrom M, A ula P, N ikoskelainen $\mathrm{E}$ et al. A mitochondrial mutation at nt 9101 in the ATP synthase 6 gene 
associated with deficient oxidative phosphorylation in a family with Leber hereditary optic neuroretino pathy. Am J Hum G enet 1995; 56:1238-1240. 30. Johns DR, N eufeld MJ. Cytochrome-c oxidase mutations in Leber hereditary optic neuropathy. Biochem Biophys Res Commun 1993;196: 810-815.

31. Howell N, Halvorson S, Burns J, McC ullough DA Poulton J. W hen does bilateral optical atrophy become Leber hereditary optic neuropathy? Am J Hum Genet 1994; 53:959-963.

32. Jun $A S$, Brown MD, W allace DC. A mitochondrial DNA mutation at nucleotide pair 14459 of the NADH dehydrogenase subunit 6 gene associated with maternally inherited Leber hereditary optic neuropathy and dystonia. Proc N atl Acad Sci USA 1994; 91:6206-6210.

33. Holt IJ, Harding AE, Petty RKH, Morgan-Hughes JA. A new mitochondrial disease associated with mitochondrial D N A heteroplasmy. Am J Hum Genet 1990; 46:428-433.

34. Santorelli FM, Shanske S, Macaya A, D evivo DC, Dimauro S. The mutation at $\mathrm{Nt} 8993$ of mitochondrial DNA is a common cause of Leighs syndrome. Ann N eurol 1993; 34:827-834.

35. Tatuch Y Robinson BH. The Mitochondrial DN A Mutation at 8993 Associated with N ARP Slows the rate of ATP synthesis in isolated lymphoblast mitochondria. Biochem Biophys Res Commun 1993; 192:124-128.

36. D evries DD, Vanengelen BG M, G abreels FJM, Ruitenbeek W, Vanoost BA. A 2nd missense mutation in the mitochondrial ATPase- 6 gene in Leigh's syndrome. Ann N eurol 1993; 34:410-412.

37. G oto YI, N onaka I, Horai S. A mutation in the tRN A Leu (UUR) gene associated with the MELAS subgroup of mitochondrial encephaloyopathies. N ature 1990; 348:651-653.

38. Sato W, Hayasaka K, Shoji Y, Takahashi T, Takada G, Saito M et al. A mitochondrial tRNA(Leu(uur) mutation at 3256 associated with mitochondrial myopathy, encephalopathy, lactic acidosis, and stroke-like episodes (MELAS). Biochem Mol Biol Int 1994; 33:1055-1061.

39. Goto YI, N onaka I, Horai S. A new mtD N A mutation associated with mitochondrial myopathy, encephalopathy, lactic acidosis, and stroke-like episodes (MELAS). Biochim Biophys Acta 1991; 1097:238-240.

40. G oto YI, T sugane K, Tanabe Y, N onaka I, Horai S. A new point mutation at nucleotide pair 3291 of the mitochondrial tRNA(Leu(uur) $)$ gene in a patient with mitochondrial myopathy, encephalopathy, lactic acidosis, and stroke-like episodes (MELAS). Biochem Biophys Res Commun 1994; 202:1624-1630.

41. Manfredi G, Schon EA, Moraes CT, Bonilla E, Berry GT, Sladky JT et al. A new mutation associated with MELAS is located in a mitochondrial DN A polypeptide-coding gene. Neuromuscular Disord 1995; 5:391-398. 42. Shoffner JM, Lott MT, Lezza AMS, Seibel P, Ballinger SW W allace DC. Myoclonic epelipsy and ragged-red fiber desease (MERRF) is associated with a mitochondrial DNA tRN A ${ }^{\text {LYS }}$ mutation. Cell 1990; 61:931-937. 43. Silvestri G, Moraes CT, Shanske S, O h S), DiMauro S. A new mtDN A mutation in the trans-RNA(Lys) gene associated with myoclonic epilepsy and ragged-red fibers (MERRF). Am J Hum Genet 1992; 51:1213-1217. 44. Van den $O$ uweland JMW, Lemkes HHPJ, Gerbitz KD, Maassen JA. Maternally inherited diabetes and deafness (MIDD): A distinct subtype of diabetes associated with mitochondrial tRN A (Leu(uur) gene point mutation. Muscle \& N erve 1995; S3:S124-S130.

45. Zeviani M, Gellera C, Antozzi C, Rimoldi M, Morandi L, Villani F et al. Maternal inherited myopathy and cardiomyopathy: Association with mutation in mitochondrial DNA tRN A (Leu(uur). Lancet 1991; 338:143-147. 46. Silvestri G, Santorelli FM, Shanske S, W hitley CB, Schimmenti LA, Smith $\mathrm{SA}$ et al. A new mtDNA mutation in the tRNA(Leu (urr) gene associated with maternally inherited cardiomyopathy. Hum Mutat 1994; 3:37-43.

47. Taniike M, Fukushima H, Yanagihara I, Tsukamoto H, Tanakaj. Mitocondrial tRN A ${ }^{\text {lle }}$ mutation in fatal cardiomypathy. Biochem Biophys Res Commun 1992; 186:47-53.
48. C asali C, Santorelli FM, D amati G, Bernucci P, D ebiase L, D iMauro S. A novel mtDNA point mutation in maternally inherited cardiomyopathy. Biochem Biophys Res Commun 1995; 213:588-593.

49. Tanaka M, Ino H, O hno K, Hattoti K, Sato W, O zawa T et al. Mitochondrial mutation in fatal infantile cardiomyopathy. Lancet 1990;336:1452.

50. Santorelli FM, Mak SC, Vazquezacevedo M, Gonzalezastiazaran A, Ridaurasanz $C$, Gonzalezhalphen $D$ et al. A novel mitochondrial DNA point mutation associated with mitochondrial encephalocardiomyopathy. Biochem Biophys Res Commun 1995; 216:835-840.

51. Santorelli FM, Mak SC, Elschahawi M, C asali C, Shanske S, Baram TZ et al. Maternally inherited cardiomyopathy and hearing loss associated with a novel mutation in the mitochondrial tRN A(Lys) gene (G 8363A). Am J Hum Genet 1996; 58:933-939.

52. Merante F, Tein I, Benson L Robinson BH. Maternally inherited hypertrophic cardiomyopathy due to a novel T-to-C transition at nucleotide 9997 in the mitochondrial tRN A(glycine) gene. Am J Hum Genet 1994; 55:437-446

53. G oto YI, Horai S, Matsuoka T, Yoga Y, N ihei K, Kobayashi M et al. Mitochondrial myopathy, encephalopathy, lactic acidosis, and stroke-like episodes (MELAS): A correlative study of the clinical features and mitochondrial DNA mutation. Neurology 1992; 42:545-550.

54. Bindoff LA, Howell N, Poulton J, Mccullough DA, Morten KJ, Lightowlers RN et al. Abnormal RN A processing associated with a novel transfer RNA mutation in mitochondrial DNA - A potential disease mechanism. J Biol Chem 1993; 268:19559-19564.

55. Moraes CT, Ciacci F, Bonilla E, Ionasescu V, Schon EA Dimauro S. A mitochondrial transfer RNA anticodon swap associated with a muscle disease. $N$ at $G$ enet 1993; 4:284-288.

56. Prezant TR, A gapian JV, Bohlman MC, Bu XD, O ztas S, Q iu W Q et al. Mitochondrial ribosomal RNA mutation associated with both antibioticinduced and non-syndromic deafness. N at Genet 1993; 4:289-294.

57. Reid FM, Vernham GA, Jacobs HT. A novel mitochondrial point mutation in a maternal pedigree with sensorineural deafness. Hum Mutat 1994; 3:243-247.

58. Gattermann N, Retzlaff S, W ang YL, Berneburg M, Heinisch J, W laschek $M$ et al. A heteroplasmic point mutation of mitochondrial tRN $A(\text { Leu })^{(\mathrm{CUN})}$ in non-lymphoid haemopoietic cell lineages from a patient with acquired idiopathic sideroblastic anaemia. Br J Haematol 1996; 93:845-855.

59. Gamez J, Playán A, Andreu AL, Bruno C, N avarro C, Cervera $C$ et al. Familial multiple symmetric lipomatosis associated with the $\mathrm{A} 8344 \mathrm{G} \mathrm{mu-}$ tation of mitochondrial DN A. N eurology 1998; 51:258-260.

60. Moraes CT, DiMauro S, Zeviani M, Lombes A, Shanske S Miranda AF. Mitochondrial DNA deletions in progressive external ophtalmoplegia and Kearns-Sayre syndrome. N Engl J Med 1989; 320:1293-1299.

61. Hammans SR, Sweeney MG, Hanna MG, Brockington M, Morganhughes JA, Harding AE. The mitochondrial DNA transfer RNA(Leu(ur) $) A$ $>G$ (3243) mutation-A clinical and genetic study. Brain 1995; 118:721-734. 62. Seibel P, Lauber J, Klopstock T, Marsac C, Kadenbach B, Reichmann H. Chronic progressive external ophthalmoplegia is associated with a novel mutation in the mitochondrial tRNA(Asn) gene. Biochem Biophys Res Commun 1994; 204:482-489.

63. Moraes CT, Ciacci F, Bonilla E, Jansen C, Hirano M, Rao N et al. Two novel pathogenic mitochondrial DN A mutations affecting organelle number and protein synthesis-Is the transfer RNA(Leu (urr) gene an etiologic hot spot? J Clin Invest 1993; 92:2906-2915.

64. Andreu AL, Hanna MG , Reichmann H, Bruno C, Penn AS, Tanji K et al. Exercise intolerance due to mutations in the cytochrome $b$ gene of mitochondrial DNA. N Engl J Med 1999; 341:1037-1044.

65. Yoon KL, A prile JR, Ernst SG. Mitochondrial tRN AThr mutation in fatal infantile respiratory enzyme deficiency. Biochem Biophys Res Commun 1991; 176:1112-1115. 
66. Sweeney MG, Bundey S, Brockington M, Poulton KR, W iner JB, Harding AE. Mitochondrial myopathy associated with sudden death in young adults and a novel mutation in the mitochondrial DN A leucine transfer RN $A^{\text {(uur) }}$ gene. Q J Med 1993; 86:709-713.

67. Thyagarajan $D$, Shanske $S$, Vazquezmemije $M$, D evivo D Dimauro S. A novel mitochondrial ATPase 6 point mutation in familial bilateral striatal necrosis. Ann N eurol 1995; 38:468-472.

68. D emeirleir L, Seneca S, Lissens W, Schoentjes E, D esprechins B. Bilateral striatal necrosis with a novel point mutation in the mitochondrial ATPase 6 gene. Pediatr N eurol 1995; 13:242-246.

69. Morten KJ, Cooper JM, Brown GK, Lake BD, Pike D Poulton J. A new point mutation associated with mitochondrial encephalomyopathy. Hum Mol Genet 1993; 2:2081-2087.

70.- N elson I, Hanna MG, Alsanjari N, Scaravilli F, Morganhughes JA, Harding AE. A new mitochondrial DNA mutation associated with progressive dementia and chorea: A clinical, pathological, and molecular genetic study. Ann N eurol 1995; 37:400-403.

71. Rotig A, Colonna M, Bonnefont JP, Blanche S, Fischer A, Saudubray JM et al. Mitochondria DNA deletion in Pearson's marrow/pancreas sindrome. Lancet 1989; 902-903.

72. Hao HL, Bonilla E, Manfredi G, Dimauro S, Moraes CT. Segregation patterns of a novel mutation in the mitochondrial tRNA glutamic acid gene associated with myopathy and diabetes mellitus. Am J Hum Genet 1995; 56:1017-1025.

73. Zeviani M, Moraes CT, DiMauro S, N akase H, Bonilla E, Schon EA et al. Deletions of mitochondrial DNA in Kearns-Sayre syndrome. Neurology 1998; 51:1525.
74. Maassen JA, Kadowaki T. Maternally inherited diabetes and deafness: A new diabetes subtype. Diabetologia 1996; 39:375-382.

75. Casademont J, Barrientos A, Cardellach F, Rotig A, G rau JM, Montoya J et al. Multiple deletions of mtDNA in two brothers with sideroblastic anemia and mitochondrial myopathy and in their asymptomatic mother. Hum Mol Genet 1994; 3:1945-1949.

76. Triepels RH, van den Heuvel LP, Loeffen JL, Buskens CA, Smeets RJ, Rubio-Gozalbo ME et al. Leigh syndrome associated with a mutation in the N DUFS7 (PSST) nuclear encoded subunit of complex I. Ann N eurol 1999; 45:787-790.

77. Lo effen J, Smeitink A, Triepels R, Smeets R, Schuelke M, Sengers R et al. The first nuclear-encoded complex I mutation in a patient with Leigh syndrome. Am J Hum Genet 1998; 63:1598-1608.

78. Bourgerom T, Rustin P, Chretien D, Birch-Machin M, Bourgeo is M, Viegas-Pequignot $\mathrm{E}$ et al. Mutation of a nuclear succinate dehydrogenase gene results in mitochondrial respiratory chain deficiency. N at Genet 1995; 11:144-149.

79. Tiranti V, Hoertnagel K, Carrozzo R, Galimberti C, Munaro M, Granatiero $M$ et al. Mutations of SURF-1 in Leigh disease associated with cytochrome c oxidase deficiency. Am J Hum Genet 1998; 63:1609-1621.

80. Zhu ZQ, Yao JB, Johns T, Fu K, D eBie I, MacMillan C et al. SURF 1, encoding a factor involved in the biogenesis of cytochrome $\mathrm{c}$ oxidase, is mutated in Leigh syndrome. $N$ at Genet 1998; 20:337-343. 